7th International Workshop on Astronomy and

Relativistic Astrophysics (IWARA 2016)

International Journal of Modern Physics: Conference Series

Vol. 45 (2017) 1760045 (9 pages)

(C) The Author(s)

DOI: $10.1142 / \mathrm{S} 201019451760045 \mathrm{X}$

\title{
Quantum Relativistic Electron Gas Expanding in One Dimension
}

\author{
Hugo Pérez Rojas \\ Departamento de Física Teórica \\ Instituto de Cibernética Matemática y Física (ICIMAF) \\ Calle E esq 15 No. 309, Vedado, 10400 La Habana Cuba \\ hugo@icimaf.cu \\ Elizabeth Rodríguez Querts \\ Departamento de Física Teórica \\ Instituto de Cibernética Matemática y Física (ICIMAF) \\ Calle E esq 15 No. 309, Vedado, 10400 La Habana Cuba \\ Departamento de Física \\ Centro de Investigación y de Estudios Avanzados (CINVESTAV-IPN) \\ Apdo. Postal 14-740, 07000 C. México, México \\ elizabeth@icimaf.cu \\ Aurora Pérez Martínez \\ Departamento de Física Teórica \\ Instituto de Cibernética Matemática y Física (ICIMAF) \\ Calle E esq 15 No. 309, Vedado, 10400 La Habana Cuba \\ Instituto de Ciencias Nucleares \\ Universidad Nacional Autónoma de México (ICN-UNAM) \\ A. P.70-543, 04510 C. México, México \\ aurora@icimaf.cu
}

Published 15 August 2017

\begin{abstract}
Under the action of field intensities around the Schwinger critical field, a dense electron gas behaves as unidimensional, exerting strong pressure along the applied field. We suggest a model for maintaining the magnetic field self-consistently, by assuming spin parallel pairing leading to a partial bosonization of the electron gas, which is described by a charged vector boson field, able to experience condensation, leading to a ferromagnetic behavior. Our aim is to suggest a possible quantum relativistic self-magnetized jet model. High frequency photons will be deviated also along paths parallel to the external field, leading to a model for a jet. Any addition of matter and/or energy to the electron
\end{abstract}

This is an Open Access article published by World Scientific Publishing Company. It is distributed under the terms of the Creative Commons Attribution 4.0 (CC-BY) License. Further distribution of this work is permitted, provided the original work is properly cited. 
system, would contribute to increase the kinetic energy along the magnetic field axis, an the jet may extend for long distances.

Keywords: Magnetized Fermion Gas, Magnetic Fields, Astrophysical Jets.

PACS numbers: 05.30.Fk, 12.20.Ds, 98.58.Fd

\section{Introduction}

Although it is sometimes underestimated by some astrophysicists, quantum effects leading to macroscopic consequences may be very important in astroparticle physics. Black hole theory is an example, although the the Chandrasekhar limit was probably the first one. For an electron density $N$ if one define $q=\lambda / d$, where $\lambda$ is the de Broglie thermal wavelength and $d=N^{-1 / 3}$ the average electron separation, quantum degeneracy become manifest for $q \gtrsim 1$, that is, for $N \geq 10^{30}$, degeneracy may exist even for $T \sim 10^{7} \mathrm{~K}$. In the white dwarf problem, the system equilibrium and stability depends on the interplay of the electron gas pressure with the pressure generated by the external gravitational field, due mainly to baryon mass, leading to a quantum limit to the stabled star mass as $M<N_{\max } M_{B}$, where $N_{\max }=$ $\left(m_{P} / m_{B}\right)^{3} \sim 10^{57}$.

The electron-positron dynamics in a magnetic field is characterized by an anisotropic spectrum

$$
E_{n, p_{3}}=c \sqrt{p_{3}^{2}+[2 e B(n+1 / 2) \pm e B] \hbar / c+m^{2} c^{2}}
$$

where $n=0,1, \ldots$ are Landau quantum numbers. This has as implication that for magnetic fields strong enough the anisotropic electron dynamics lead to a significant anisotropic electron thermodynamics (pressures are anisotropic) and an anisotropic photon propagation (see Refs. ${ }^{1-3}$ ). This is because the magnetic field breaks the symmetry of vacuum. As we assume an homogeneous constant magnetic field, the resulting symmetry is rotational invariant around the direction of $\mathbf{B}$.

Let us remind that the longitudinal pressure is $p_{3}=-\Omega$. In an earlier paper ${ }^{4}$ it was shown that in degenerate conditions of the electron gas, the transverse pressure to the magnetic field $\mathbf{B}$,

$$
p_{\perp}=-\Omega-B \mathcal{M},
$$

where $\mathcal{M}$ is the magnetization, may vanish for fields large enough. The effect is easily to understood since, as all electrons are confined to the LLL, the quantum average of their transverse momentum vanishes. We are assuming an electron chemical potential $\mu \gg T$. The maximum occupied Landau quantum number $n_{M}$ is given by the inequality

$$
n_{M} \leq\left(\theta^{2}-1\right) / 2 b,
$$

where $\theta=\mu / m, b=B / B_{c}$, in which the Schwinger critical field

$$
B_{c}=m^{2} c^{3} / e \hbar \sim 4.4 \times 10^{13} G .
$$


Thus the confinement to the LLL is possible only if

$$
\left(\theta^{2}-1\right) / 2 b<1 \text {, }
$$

which define the compatible range of values of $b$ and $\theta$ as given by

$$
\theta^{2}<2 b+1 \text {. }
$$

The expression for the magnetization in the degenerate limit is

$$
\mathcal{M}=\frac{\alpha B_{c}}{4 \pi^{2}}\left(\theta \sqrt{\theta^{2}-1}-\ln \left(\theta+\sqrt{\theta^{2}-1}\right)\right) .
$$

The system is paramagnetic, since $\mathcal{M}>0$ and it is a function of the chemical potential $\mu$, which is in turn a function $N$ and $B$ (see below). As

$$
\mathcal{M}=-\partial \Omega / \partial B
$$

it is general

$$
\Omega=-\int \mathcal{M}(B) d B+f,
$$

where $f$ is independent of $B$. In the present approximation, where only the LLL is occupied, it is

$$
\Omega=-\mathcal{M} B
$$

and in consequence

$$
p_{\perp}=-\Omega-B \mathcal{M}=0 .
$$

This corresponds to densities of order of the ones expected for white dwarfs. The electron gas becomes a one dimensional system along the field ${ }^{\mathrm{a}} \mathbf{B}$. Critical fields $b \sim 1$ cannot exist in stable white dwarfs.

The one-dimensional electron behavior is related to the following physical facts ${ }^{2}$ : the quantity $S_{\phi}=c \hbar / e B$ can be considered as the quantum of area corresponding to a flux quantum $\phi=c \hbar / e$ for a field intensity $\mathbf{B}$. Thus, by increasing $B, S_{\phi}$ decreases. As a consequence, the spread of the electron and positron wave functions decrease exponentially with $B$ in the direction orthogonal to the field since they depend on the transverse coordinates as $e^{-\xi^{2}}$ where $\xi^{2}=x_{\perp}^{2} / S_{\phi}$. Actually, such behavior is the same for charged vector bosons, whose ground state wavefunction is expressed in terms of Hermite fuctions depending from $\xi^{2}$.

We conclude that for fields near the critical value, the spread of the wave function is concentrated in an area of order of a squared Compton wavelength. As the motion of electrons and positrons along the magnetic field is free, from a macroscopic point of view they have essentially a one-dimensional behavior.

We must point out here that there is a negative (Casimir-like) pressure in magnetized vacuum ${ }^{1}$ in the direction orthogonal to the field $\mathbf{B}$. It acts as a compression

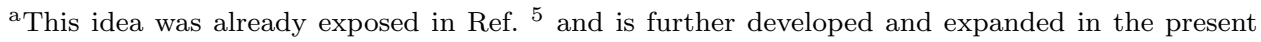
paper. 
and as a consequence, vacuum is also compressed inward in the direction orthogonal to $\mathbf{B}$, as it occurs for observable gas of electrons in a magnetic field. The pressures are also $p_{3}$ parallel to $\mathbf{B}$, and $p_{\perp}=p_{3}-B \mathcal{M}$ orthogonal to it.

\section{Electron Speeds at Critical Fields}

Let us assume the electron gas at a sufficiently high density $N$ and strong magnetic field $B$ such that the system is confined to the lowest Landau level (LLL), so that from (1)

$$
E_{0, p_{3}}=c \sqrt{p_{3}^{2}+m^{2} c^{2}}
$$

Let us name

$$
E_{F}=\sqrt{\mu^{2}-m^{2} c^{4}}=m c^{2} \sqrt{\theta^{2}-1}
$$

the longitudinal Fermi energy. We can write then the electron density $N$ as

$$
N=e B E_{F} /\left(2 \pi \hbar^{2} c^{2}\right) \text {. }
$$

By keeping $B$ constant, if $N$ grows, the longitudinal density, which is proportional to the Fermi momentum $p_{F}=E_{F} / c=m c \sqrt{\theta^{2}-1}$ also increases. For instance as $\theta>1$, and proceeding classically by equating

$$
p_{F}=m v_{F} / \sqrt{1-\left(v_{F} / c\right)^{2}},
$$

we get the speed

$$
v_{F}=\sqrt{\theta^{2}-1} c / \theta
$$

For instance if $\theta=\sqrt{5}$, the increase of momentum leads to $v_{F}=\sim 0.9 c$. Quasiluminal speeds occur for densities such that $\theta>2$ and $b \gtrsim 2$, (magnetic fields $\left.B \gtrsim 2 B_{c}\right)$. At fields of that order, it is increased the probability of pair creation out of $\gamma$ rays of energy $\omega \geq 2 m c^{2}$, which are deviated and forced to move along $B_{c}$ (see below). Similarly, if $E_{F}$ is constant, an increase in the density $N$ increases $B$, that is, the transverse density given by the flux quanta density.

\section{The Arising of a Ferromagnetic Phase}

The assumption of a free very dense electron gas is an oversimplification. The electrons interact among themselves and with the surrounding ions. We search for an effective ferromagnetic model which must be energetically more favourable than the original free electron gas, by assuming parallel spin-spin coupling, which as different from Cooper pairs lead to a vector bosonization of a significant amount of the total electron system. Such interaction is favoured due to having a large number of electrons with the same spin $s$ and momentum $p_{3}$, which is accounted for by the quantum degeneracy factor $e B / \hbar c$ having dimension of surface density $L^{-2}$. It leads to the system to have a large number of particles sharing the same quantum numbers, except the center of the orbit quantum number $x_{0}$, the energy spectrum being 
degenerate with regard to it. The degeneracy factor implies that at any value of momentum we may consider a shell in momentum space containing a large amount of particles, orthogonal to the z-axis. For instance, if $B \sim B_{c} \sim 4 \times 10^{13} \mathrm{G}$, the surface density orthogonal to $p_{3}$ is $e B / \hbar c \sim 10^{20} \mathrm{~cm}^{-2}$.

We may conceive the energy of the magnetized degenerate Fermion system, as composed from the contribution of successive shells, corresponding to increasing values of momentum between the two values $p_{3}= \pm p_{F}$, as given by the expression

$$
U=\frac{e B}{\hbar c} \int_{-p_{F}}^{p_{F}} n\left(E\left(p_{3}\right)-\mu\right) E\left(p_{3}\right) d p_{3},
$$

where in the present degenerate case $n\left(E\left(p_{3}\right)-\mu\right)$ is the step function. If we consider the momentum shell of width $\delta p_{3}$ around $p_{3}=0$, the corresponding energy is

$$
\delta U=\frac{e B}{\hbar c} m c^{2} \delta p_{3} .
$$

If there is a coupling of electron pairs in some subsystem of the whole system, the paired particles in such subsystem behave as a vector boson field. The pairs would carry charge $2 e$, and total spin $s=1\left(m_{s}= \pm 1\right)$, and their effective mass, would be smaller than twice the electron mass due to the vector coupling

$$
M=\sqrt{4 m^{2}-2 e B \hbar / c^{3}},
$$

(see below). The bosonization in presence of the magnetic field, leads to a ground state of lower energy, the gap being proportional to the magnetic field intensity. The larger the gap, the greater the magnetic moment of the paired electrons, and consequently, the magnetization of the system, which for an appropriate tuning, leads to a ferromagnetic behavior: the magnetic field can be maintained self-consistently.

For particles moving with nonzero momentum $p_{3}$ we can perform a Lorentz boost to a frame at a speed such that the transformed momentum is zero (For instance, for the Fermi surface, as $\mu_{F}=\theta m c^{2}, p_{F}$ are the basic relativistic variables, the speed for the boost is $\left.v_{F}=p_{F} c^{2} / \mu\right)$. Thus the new zero momentum surfaces are such that close to them particles are paired with opposite momentum $\pm p_{3}$, having also opposite helicities, since their (common) spin projection is $s=1 / 2$. As pointed out previously, we are assuming pair coupling resembling Cooper pairs, but with parallel spins and magnetic moments. Thus, we assume them as a composite vector particle of charge $2 e$ and mass $M \lesssim 2 m$, from which the system may search for a ground state having a gap with regard to the ground state of the original Fermion system. The coupled pairs have total momentum zero, and by assuming all particles at the LLL, we get a condensate of particles having effective mass $M$. The original ground state is decreased, due to the gap $-2 e B \hbar / c^{3}$. Notice that the gap is the same for all reference frames parallel to $B$ since $B$ is a pseudovector coming from the external electromagnetic field tensor whose component along the $z$ axis does not change under Lorentz transformations along it. On the other hand, in another paper of the present proceedings ${ }^{6}$, a deduction of the $e^{+}-e^{-}$neutral paired system (with similar effective mass) spectrum is given. 
Thus, we assume a model of electron pairs coupling with $\operatorname{spin} s=1$, leading to a ground state having a gap with regard the free electron ground state. This means a bosonization and consequently, Bose-Einstein condensation, leading to a ferromagnetic behavior. That is, the system may be treated as a charged vector boson field, and we follow the formalism used in previous references ${ }^{4,7}$. As a consequence of condensation the system would behave as ferromagnetic and under the action of an external field $H$, a magnetization $\mathcal{M}$ arises, leading us to define a microscopic magnetic field $B=H+4 \pi \mathcal{M}$. Due to the positive character of $\mathcal{M}$ one could equate $B \sim 4 \pi \mathcal{M}$, or $H \ll 4 \pi \mathcal{M}$ i.e., the microscopic magnetic field be produced by self-magnetization.

In Ref. ${ }^{8}$ we assumed the present relativistic vector boson spectrum as

$$
\epsilon_{0}\left(p_{3}\right)=\sqrt{p_{3}^{2} c^{2}+(2 m)^{2} c^{4}-2 e B \hbar c},
$$

for the Landau ground state $n=0$, and concluded that the effective magnetic moment in the ground state is $\mathrm{m}=e \hbar / M c$.

In Ref. ${ }^{7}$ we have shown that Bose-Einstein condensation, in the sense of having a large population in the Landau ground state, is achieved for very low momentum states of a gas of charged vector particles in presence of a strong magnetic field. We got in a small neighborhood of $p_{3}=0$,

$$
N_{0}=\frac{2 e B T}{4 \pi \hbar^{2} c} \frac{2 \mu_{b}}{\sqrt{M^{2} c^{4}-\mu_{b}^{2}}} \sim \frac{2 e B T}{4 \pi \hbar^{2} c} \sqrt{\frac{2 M}{-\mu_{b}^{\prime}}},
$$

where $\mu_{b}<M$ is the bosonic chemical potential, $\mu_{b}^{\prime}=\mu_{b}-M c^{2}$, and $N=N_{0}+\delta N$ and $\delta N$ is the density in the interval $\left[p_{0}, \infty\right]$. Actually as $\mu_{b}^{\prime} \rightarrow 0, N_{0} \rightarrow N$ and $\delta N$ is very small. We get then

$$
\mu_{b}^{\prime} \simeq-e^{2} B^{2} T^{2} M /\left(2 \pi^{2} N^{2} \hbar^{4} c^{2}\right)
$$

We observe that $\mu_{b}^{\prime}$ is a decreasing function of $T$ and vanishes for $T=0$, where the "critical" condition $\mu_{b}=M c^{2}$ is reached. As shown in Ref. ${ }^{7}$ in that limit the Bose-Einstein distribution degenerate in a Dirac $\delta$ function, which means to have all the system in the ground state $p_{3}=0$. From (5) we may write the thermodynamic potential and the magnetization, respectively, as

$$
\Omega=\frac{e B T}{2 \pi \hbar^{2} c} \sqrt{M^{2} c^{4}-\mu_{b}^{2}}, \quad \mathcal{M}=-\frac{\partial \Omega}{\partial B} \simeq \frac{e N_{0} \hbar}{M c}=N_{0} \mathrm{~m} .
$$

One can state the condition for self-magnetization, by writing $H=B-4 \pi \mathcal{M}=0$

$$
B=4 \pi \mathcal{M}=4 \pi e N_{0} \hbar /(M c) .
$$

Let us assume that $N \sim 10^{32}$. Then $\mathcal{M} \sim 10^{12}$ and $B \sim 10^{13} G$. The condition for self-magnetization is satisfied. The system becomes a giant magnet, whose stability is determined by the transverse pressure condition $P_{\perp}=-\Omega-B \mathcal{M}$ of the whole system. However, the estimation of the value of $\Omega$ would depend on the fraction of electrons paired. Let us name $N_{u}, N_{p}=N-N_{u}$ respectively the density of 
unpaired and paired electrons. If $N_{u} \sim N_{p}$. Then the dominating pressure comes from the (unpaired) electron gas contribution, $\Omega \sim N m c^{2} \sim 10^{26}$ (we assume $\mu$ of order $m c^{2}$ ), which is very close to $B \mathcal{M} \sim 10^{25}$. Thus, one can affirm that in the region $N \sim 10^{32}-10^{33}$, the bosonized ferromagnetic system may be maintained self-consistently, and inside a star, lead to a collapse. If, however, $N_{u} \ll N_{p}$ so that the dominant pressure comes from the paired gas, as $\Omega$ is positive, its contribution to pressure is negative. The stability requires from a Fermion background.

There is another point to be considered when $e B \hbar / c^{3}$ approaches to $(2 m)^{2}$. As $M$ decreases with increasing $B$, the magnetization $\mathcal{M}$ increases with $B$, and would diverge for $M \rightarrow 0$. For $e B \hbar / c^{3}$ close enough to $(2 m)^{2}$ one expects the main contribution to $B$ be produced by $\mathcal{M}$. We get an equation ${ }^{4}$ for the self consistent magnetization. Let us write $2 b=x^{2}$ where $0 \leq x \leq 1$. For $x=1$, we have the critical field $B_{C}=(2 m)^{2} c^{3} / 2 e \hbar \simeq 8.82 \times 10^{13} \mathrm{G}$. Then we can write $M=2 m \sqrt{1-x^{2}}$, and

$$
x^{2} \sqrt{1-x^{2}}=A=8 \pi e^{2} \hbar^{2} N /\left[(2 m)^{3} c^{4}\right] .
$$

By simple inspection we find that it has real solutions only for $A<2 \sqrt{3} / 9=A_{1}$. This means that $N \leq 10^{32}$. By solving the cubic equation (9), we find that for $A \ll 1$, these real solutions are $x_{1}=\sqrt{A+A^{2} / 2}$ and $x_{2}=\sqrt{1-A^{2}}$. The first solution means that $B$ increases with increasing $N$, up to the value $B_{\max }=2 / 3 B_{C}$. In the second solution $B$ decreases for growing $N$, and its limit for $N \rightarrow 0$ being $B_{C}$, and is not interesting to us in the present context.

From all the previous reasoning we conclude that inside a star an electron system, can be hardly stable at fields of order $B_{c}$. In principle, such fields can be maintained self-consistently, but the possibility of a collapse is highly increased: the one-dimensional world created by the magnetic field is completely unstable. However, such structures at critical field values may lead to jets coming outside cosmic objects, with the longitudinal pressure dominating along the self-consistently maintained magnetic field. Even radiation would be forced to move in straight line, as discussed below. It is important to remark here that that the jet electrons ${ }^{9}$ exerting the pressure parallel to $\mathbf{B}$ would have helicity L, those exerting the pressure antiparallel to $\mathbf{B}$ have helicity $\mathbf{R}$.

\section{The Role of Photons}

Let us now consider photons that are created inside the cosmic object or from outside it. If the body is a star its magnetic field we assume to grow with rotational symmetry as we aproach to the symmetry axis. Photons moving in a region of increasing magnetic field are decreased in their momentum orthogonal to $B$, and for very large fields (critical and supercritical, $b \gtrsim 1$ ) they are confined to move parallel to $B$, as was shown in detail in Refs. ${ }^{2,5}$. For frequencies $\omega \simeq 2 m c^{2} / \hbar$ and $k_{\|} c<\omega$, the dispersion equation for the second mode may be written ${ }^{10}$, if the polarization operator is expressed as a sum over Landau levels $n, n^{\prime}$ of the 
virtual electron-positron pairs, in terms of the dominant term $n=n^{\prime}=0$, (we call $z_{1}=\left(k_{\|}^{2} c^{2}-\omega^{2}\right) \hbar^{2}, z_{2}=k_{\perp}^{2} c^{2} \hbar^{2}$ which are relativistic invariant quantities) as

$$
z_{1}+z_{2}=2 \alpha e B m \hbar c^{3} e^{-z_{2} /(2 e B \hbar c)} / \sqrt{z_{1}+4 m^{2} c^{4}} .
$$

Notice that its limit for $\mathbf{k} \rightarrow \mathbf{0}$ is $\omega \neq 0$. Actually, it describes a massive vector boson particle closely related to the electron-positron pair.

The Eq. (10) has solutions found by Shabad as those of a cubic equation. One can estimate its behavior very near $z_{1}=-4 m^{2} c^{4}$, by assuming $z_{1}=-4 m^{2} c^{4}+\epsilon$ and $z_{2}=4 m^{2} c^{4}-\epsilon$, where $\epsilon$ is a small quantity. One can obtain approximately

$$
\omega=\sqrt{k_{\|}^{2} c^{2} \hbar^{2}+4 m^{2} c^{4}-\left(2 \alpha e B m \hbar c^{3} e^{-2 m^{2} c^{3} / e B \hbar}\right)^{2 / 3}} .
$$

Thus, the transverse momentum of the original photon is absorbed by the magnetized medium, the resulting quasi-particle being deviated to move along the field as a vector boson of mass

$$
m_{e f f}=\sqrt{4 m^{2}-e B_{c}\left(2 \alpha b e^{-2 / b}\right)^{2 / 3} \hbar / c^{3}} .
$$

For slightly larger energies such that $z_{1}=-4 m^{2} c^{4}-\epsilon$, and $b \sim 1$, they decay in observable electron-positron pairs, and the polarized vacuum becomes absorptive.

Notice that the massive photon bears an effective mass $m_{e f f}$ which was obtained in first order of perturbation theory. For frequencies larger than $2 m c^{2}$ (first pair creation threshold), we expect a similar spectrum. This means that for magnetic fields of order $2 B_{c}$, positronium is formed, and although it has a very small lifetime, in an electron-positron medium Pauli's principle may forbid its decay in some electron environment, and would preserve it with some stability, so that it may contribute also to the self-consistent magnetic field.

The final picture of electrons and photons is a magnetized jet: a one dimensional expanding object composed by electrons, high energy photons and some amount of matter pushed by them. Any addition of energy to the electron system, could contribute only to increase its kinetic energy along the magnetic field axis, an the jet may grow in this way indefinitely.

\section{Acknowledgments}

The authors thanks to the Abdus Salam ICTP OEA Office for support under Net35. ERQQ has been also supported by a TWAS-CONACYT 2015 postdoctoral fellowship. A.P.M thanks Consejo Nacional de Ciencia y tecnologia (CONACYT) for the support with the sabbatical Grant 264150 at ICN-UNAM, Mexico, where this work was developed.

\section{References}

1. H. Pérez Rojas, E. Rodriguez Querts, Int. J. Mod. Phys. A 21, 3761 (2006).

2. H. Pérez Rojas, E. Rodríguez Querts, Eur. Phys. J. C 74, 2899 (2014). 
3. H. Pérez Rojas, E. Rodríguez Querts, Phys. Rev. D 79, 093002 (2009).

4. M. Chaichian, S. S. Masood, C. Montonen, A. Pérez Martínez, and H. Perez Rojas Phys. Rev. Lett. 84, 5261(2000).

5. H. Pérez Rojas, E. Rodríguez Querts, and A. Pérez Martínez, Astron. Nachr. 336, 885 (2015).

6. G. Quintero Angulo, A. Pérez Martínez, and H. Pérez Rojas, Condensation of neutral vector bosons with AMM, Int. J. Mod. Phys.: Conference Series, XX, XXXXX (2017).

7. H. Pérez Rojas, Phys. Lett. B 379, 148 (1996).

8. A. Pérez Martínez, H. Pérez Rojas, and H. J. Mosquera Cuesta, Chin. Phys. Lett.2004 21, 2117 (2004).

9. J. L. Acosta Avalo and H. Pérez Rojas, Nucl. Phys. B 909, 230-242 (2016).

10. A. E. Shabad, Annals Phys. 90, 166 (1975). 